sexual differentiation and pituitary hormone secretion. The final chapter concludes that the brain catecholamine systems appeared very early in evolution and seem to play important roles in many brain functions but emphasizes the major differences in anatomy and distribution between different vertebrate classes and points out the relative lack of data on the noradrenergic and adrenergic systems as compared to the dopamine system.

The emphasis throughout is on the presynaptic catecholamine systems; postsynaptic receptors are rarely mentioned except in regard to dopamine receptors in birds and in the basal ganglia and cortex of mammals.

Researchers working on the anatomy of catecholamine systems in various vertebrates should find this a very useful reference book. Of particular interest to this reviewer was the emphasis on differences in anatomy and distribution in various classes or, within mammalians, in various species.

\section{Edith G. McGeer} Vancouver, British Columbia

ATLAS OF ADULT ELECTROENCEPHALOGRAPHY. 1994. By Warren T. Blume and Masako Kaibara. Published by Raven Press. 585 pages. $\$ \mathrm{C} 129.00$

In an era when we daily receive advertisements for new publications, "advances" and "updates" it may come as a surprise that someone has undertaken the task of producing a new atlas of one of the oldest investigative techniques in neurology. However, electroencephalographers familiar with the currently available (relatively few) atlases will recognize why Blume and Kaibara's volume is a welcome addition

The ratio of illustrations (516) to pages (585) and number of references (58) testifies that this is an atlas, not a textbook, of EEG. The topics covered include artifacts, normal phenomena, interictal epileptiform abnormalities, seizures, nonepileptiform abnormalities and coma.

The illustrations have been reproduced with uncommon clarity. All of the EEGs are derived from scalp recordings using 16-18 channels (although many examples show only the relevant 8 or more channels).

The captions with each illustration are detailed, informative and reflect not only the authors' mastery of EEG interpretation but also the techniques of how to teach EEG. Emphasis is placed on a thorough understanding of basic EEG principles, particularly polarity. Considerable attention is given to "problem solving" EEG phenomena; i.e., what is the state of the patient? what is the polarity of the discharge? what is the electrical field of the discharge? could it be an artifact? if so, what type of artifact?

The presentation usually starts with "textbook" illustrations of EEG phenomena that are followed by increasingly complicated and atypical examples (including those with artifact and montages that display the waveforms in a sub-optimum manner). Some readers might object to this apparent redundancy but the pedagogic value and resemblance to "real world" EEG cannot be denied.

Criticisms? Very few! Examples of the difficulties in EEG interpretation of pseudoseizures would have been useful. The majority of EEGs have adequate space but the occasional illustration looks a bit cramped. I suspect that had a large format approach been adopted (such as Blume's previously published Atlas of Pediatric Electroencephalography) the price would have been prohibitive. EEG from intracranial monitoring is not included despite the authors' extensive experience in this field. Perhaps a few examples of simultaneous extracranial-intracranial EEGs would give an appreciation of what is recorded, and missed, by scalp electrodes.

How should this book be used? In the preface to this atlas the authors state that it is intended to complement a textbook of EEG (Current Practice of Clinical Electroencephalography; edited by D. Daly and T.A. Pedley). The novice should read this atlas slowly and carefully; this is a book designed to enhance pattern recognition that cannot be gleaned from a textbook. To paraphrase Osler, to study the phenomena of EEG without books is to sail an uncharted sea, while to study books without reading EEG records is not to go to sea at all. The experienced electroencephalographer will also find many subtleties of interest (at least I did).

This book should be in all EEG laboratories, irrespective of the presence of a training program. Dr. Blume has superior EEG reading abilities and is an outstanding teacher of these skills. I learned EEG from him during hours of rigorous reading sessions. This atlas is "the next best thing to being there." I think this is the best available EEG atlas and give it a very enthusiastic "thumbs up!"

Mark Sadler

Halifax, Nova Scotia

THE EPILEPSY HANDBOOK. THE PRACTICAL MANAGEMENT OF SEIZURES. 1994. 2nd Edition. By R.J. Gumnit. Published by Raven Press. 194 pages. $\$ C 49.00$

The rapid changes in the field of epilepsy required that this second edition, more than a decade after the first one, had to be largely rewritten. The author has succeeded in covering the management of seizures in its broadest sense in a concise, logical fashion.

After defining epilepsy and seizures, the author gives ample attention to the management of the first seizure. The author states that only $50 \%$ of patients are diagnosed in the first six months and that it takes five years following the first seizure before $85 \%$ of the patients will have been diagnosed with epilepsy. This indicates that much work still has to be done, informing primary care physicians, other health care providers and patients.

Very practical guidelines for the use of conventional and novel anti-epileptic drugs are given. Special chapters deal with pregnancy and teratogenicity, status epilepticus, surgical treatment and the management of children and infants with epilepsy. Differential diagnosis and the criteria for referral to epileptologists and epilepsy centers are discussed in detail.

The information on the social aspects of epilepsy including insurance, driving, the law and recreation is very helpful for physicians, patients and their relatives.

The information is accurate and very well presented, with little pearls reflecting the wide clinical experience of the author. "Clinical Vignettes" is a chapter where the author-clinician clarifies difficult concepts by presenting brief interesting case reports.

This book is published in the United States and therefore the Canadian reader will not find information on the slow-release form of carbamazepine (Tegretol CR), clobazam (Frisium) and vigabatrin (Sabril), anti-epileptic drugs that in Canada have become a mainstay in the management of the most common seizure types. These drugs are presently not widely available in the United States. Nevertheless, I highly recommend this book to primary care physicians, neurologists and people who take care of patients with epilepsy.

Elout Starreveld Edmonton, Alberta 